\title{
Final Report on Clinical Outcomes and Tumor Recurrence Patterns of a Pilot Study Assessing Efficacy of Belinostat (PXD-101) With Chemoradiation for Newly Diagnosed Glioblastoma
}

\section{Karen Xu}

Emory University School of Medicine

Karthik Ramesh

Emory University School of Medicine

Vicki Huang

Emory University School of Medicine

Saumya S Gurbani

Emory University School of Medicine

J Scott Cordova

Emory University School of Medicine

Eduard Schreibmann

Emory University School of Medicine

Brent D Weinberg

Emory University School of Medicine

\section{Soma Sengupta}

Emory University School of Medicine

Alfredo D Voloschin

Emory University School of Medicine

Matthias Holdhoff

Johns Hopkins University

Peter B Barker

Johns Hopkins University

Lawrence R Kleinberg

Johns Hopkins University

Jeffrey J Olson

Emory University School of Medicine

Hui-Kuo G Shu

Emory University School of Medicine

Hyunsuk Shim ( $\square$ hshim@emory.edu )

Emory University School of Medicine https://orcid.org/0000-0001-8313-2612 


\section{Research Article}

Keywords: Glioblastoma, Histone deacetylase, Epigenetic drug, Radiation sensitizer, Magnetic resonance spectroscopy

Posted Date: June 29th, 2021

DOI: https://doi.org/10.21203/rs.3.rs-650923/v1

License: (c) (1) This work is licensed under a Creative Commons Attribution 4.0 International License. Read Full License

Version of Record: A version of this preprint was published at Tomography on March 3rd, 2022. See the published version at https://doi.org/10.3390/tomography8020057. 


\section{Abstract}

\section{Purpose}

Glioblastoma (GBM) is highly aggressive with poor prognosis. Belinostat is a histone deacetylase inhibitor with blood-brain barrier permeability, anti-GBM activity, and potential to enhance chemoradiation. The purpose of this clinical trial was to assess the clinical efficacy of combining belinostat with standard-of-care therapy for GBMs.

\section{Methods}

13 patients were enrolled in each of the control and belinostat cohorts. The belinostat cohort was given a belinostat regimen (500-750 mg/m $\mathrm{m}^{2} 1 \mathrm{x} /$ day $\times 5$ days) every 3 weeks (weeks 0,3 , and 6 of RT). All patients received temozolomide and radiation therapy (RT). RT margins of $5-10 \mathrm{~mm}$ were added to generate clinical tumor volumes and $3 \mathrm{~mm}$ added to create planning target volumes. Patient outcomes included progression-free survival, overall survival (OS), and recurrence pattern analysis of enhancing tumor (rGTV).

Results

Median OS was 15.8 months for the control cohort and 18.5 months for the belinostat cohort $(p=0.53)$. The rGTVs in the control cohort occurred in areas that received higher radiation doses than that in the belinostat cohort. For those belinostat patients who experienced out-of-the field recurrence, tumors were detectable by spectroscopic MRI before RT.

Conclusion

The median OS was slightly longer for the belinostat cohort than the control cohort but not statistically significant. Recurrence analysis suggests better in-field control with belinostat, suggesting a radiosensitizing effect. This study highlights the potential of belinostat as a synergistic therapeutic agent for GBM treatment. It may be particularly beneficial to combine this radio-sensitizing effect with spectroscopic MRI-guided radiation therapy.

\section{Introduction}

Glioblastoma (GBM) is the most common primary brain tumor in adults. It is highly aggressive and associated with poor prognosis despite multimodal treatment. The current treatment paradigm consists of maximal safe neurosurgical resection followed by radiation therapy (RT) with concurrent and adjuvant temozolomide (TMZ). This regimen consists of focal RT to 60 Gy over 6 weeks with concurrent TMZ given at $75 \mathrm{mg} / \mathrm{m}^{2} /$ day followed by adjuvant TMZ given at $150-200 \mathrm{mg} / \mathrm{m}^{2} /$ day for days 1 to 5 of a 28day cycle for up to 12 months. Despite comprehensive treatments, median overall survival (OS) remains at 16 months [1-6]. 
Histone deacetylases regulate a wide variety of cellular functions and play a role in re-differentiation of various tumors. Histone deacetylase inhibitors (HDACi) have been shown to improve outcomes for patients with gliomas [7]. In 2006, suberanilohydroxamic acid (SAHA), a first-generation HDACi which targets multiple class I and class II HDAC family members, became the first HDACi to receive FDA approval for advanced cutaneous T-cell lymphomas [8].

Belinostat (PXD-101, Acrotech Biopharma, LLC, East Windsor, NJ), a later generation pan-HDACi, improves upon SAHA with increased potency and BBB penetration $[9,10]$. Belinostat received FDA approval for patients with relapsed/refractory peripheral T-cell lymphoma in 2014 [11]. Preclinical investigations have shown antitumor effects in orthotopic glioma animal models [12]. This suggests that development of a potent HDACi capable of penetrating the BBB has the potential to improve outcomes of patients with GBM. In addition to anti-tumor effects, we reported that belinostat restores the bottle neck enzyme levels of normal brain metabolites, $\mathrm{N}$-acetylaspartate (NAA) and myo-inositol, in vitro to a greater extent than other HDACis [12]. Furthermore, spectroscopic magnetic resonance imaging (sMRI), a quantitative imaging technique that assesses metabolic responses in vivo without any contrast agent injection, has been used to show a GBM patient with an IDH mutation (without MGMT promoter hypermethylation) exhibited remarkable response to belinostat combined with standard chemoradiation therapy [13]. In that case restoration of NAA, creatine and myo-inositol, reached the levels of healthy subjects.

We previously reported interim PFS and sMRI findings in GBM patients receiving belinostat $[12,13]$. With 50 months of median follow-up, we report the final clinical outcomes and tumor recurrence patterns of patients enrolled in this study.

\section{Methods And Materials}

Patients with newly diagnosed GBM were enrolled in either the control or treatment arm of the Institutional Review Board (IRB)-approved clinical trial at Emory and Johns Hopkins (ClinicalTrials.gov ID NCT02137759). Patients on the treatment arm received intravenous belinostat (Acrotech Biopharma, LLC, East Windsor, $\mathrm{NJ}$ ) as an investigational therapeutic.

This was not a randomized study and patients were serially enrolled into the control arm from 2014 to 2015 and then the belinostat treatment arm from 2015 to 2018. All patients, if surgical candidates, underwent maximal safe tumor resection before enrolling in the study. Patients in both arms received standard treatment including daily TMZ $\left(75 \mathrm{mg} / \mathrm{m}^{2}\right)$ and focal radiation doses of 51 Gy to T2/FLAIR abnormality (GTV1) and 60 Gy to the resection cavity/residual contrast-enhancing (CE) tissue (GTV2) on T1-weighted contrast-enhanced MRI (T1w-CE). Margins of 5 to $10 \mathrm{~mm}$ were added to generate clinical tumor volumes (CTVs) and 3 to $5 \mathrm{~mm}$ to generate planning treatment volumes (PTVs) to account for microscopic disease spread and treatment setup uncertainty, respectively. PTV1 is the FLAIR abnormal volume receiving $51 \mathrm{~Gy}$ while PTV2 is the T1w-CE volume receiving $60 \mathrm{~Gy}$. 
In the treatment arm, patients received daily intravenous doses of belinostat for 5 consecutive days in 3 cycles, 3 weeks apart, beginning 1 week before the start of chemoradiation. The first 3 patients received $750 \mathrm{mg} / \mathrm{m}^{2}$ of belinostat; however, due to two of the patients having adverse hematologic toxicity during the course of belinostat, TMZ, and radiation, the belinostat dose was lowered to $500 \mathrm{mg} / \mathrm{m}^{2}$ for the remaining patients in the trial. Patients were followed with standard MRIs for 12 months post-treatment or until radiographic progression of disease. The study timeline for the belinostat cohort is summarized in Fig. 1. PFS is reported for patients based on time to radiologic confirmation of disease progression from the date of surgery. Data are right censored for patients who were lost to follow-up. Kaplan-Meier curves for OS were generated.

sMRI data were acquired for patients before starting treatment and four weeks post-RT. The sMRI data acquisition combined 3D echo-planar spectroscopic imaging, with GRAPPA (GeneRalizaed Autocalibrating Partial Parallel Acquisition) parallelization, and elliptical k-space encoding (TE/TR/FA = $17.6 \mathrm{~ms} / 1551 \mathrm{~ms} / 71^{\circ}$ ) on 3T MRI scanners with a 32-channel head coil array (Siemens Healthineers or Philips Healthcare) [14]. Raw data were processed using the Metabolite Imaging and Data Analysis System (MIDAS) (University of Miami) [14-16] with a nominal voxel size of $4.4 \mathrm{~mm}$ x $4.4 \mathrm{~mm} \times 5.6 \mathrm{~mm}$. Metabolite maps generated included choline (Cho), Creatine ( $\mathrm{Cr}$ ), N-acetylaspartate (NAA), as well as Cho/NAA ratio maps [17]. Metabolite and ratio maps were then imported into the Brain Imaging Collaboration Suite (BrICS) for registration with the radiation planning MRI images [18]. A new volume was created based on union of Cho/NAA elevated lesions with residual contrast-enhancing volume (excluding resection cavity) as described in our dose escalation trial scheme [18].

After completing enrollment in August of 2018, we continued to track patients to assess long-term outcomes and recurrence patterns. For each patient, T1w-CE MRIs were acquired at the radiologically confirmed progression dates and co-registered to MRIs used for RT planning. The recurrence volumes based on enhancement in T1w-CE MRIs (rGTVs) were generated by manually contouring abnormal enhancement in the progressed MRIs. Overlap statistics were calculated between rGTV and PTVs (PTV 1 and 2) as well as calculation of the minimum, maximum, and mean radiation doses that the rGTV volume received using in-house automated algorithms to perform voxel-wise dose comparisons. Radiation doses received by each volume were extracted from the clinical system using the Eclipse Scripting Application Programming Interface (ESAPI) which is built into the Eclipse treatment-planning platform (Varian Medical System, Inc). Incorporation with this platform enabled automation of our method, saving time and errors that often occur from manual data extraction, with the additional benefit of using the original measurements of the radiation dose clouds for a comparison to tumor recurrence in terms of the range and median doses received by those regions. Two patients were excluded from analysis of rGTV due to follow-up imaging being unavailable.

\section{Results}

A total of 26 patients were enrolled (13 control and 13 belinostat) with median follow-up of 50 months. Patient/tumor characteristics were similar between cohorts (Supplement Table 1); summary statistics are 
highlighted in Table 1A.

Median OS was 15.8 months for the control cohort and 18.5 months for the belinostat cohort $(p=0.53)$. Comparison of OS curves for the two cohorts are shown (Fig. 2A). 6-month PFS was $54 \%$ and $84 \%(p=$ 0.073) for the control and the belinostat cohorts, respectively, reported previously [12]. The median PFS was similar in the two groups, being 9.0 months for the control and 9.3 months for the belinostat cohorts $(p=0.75)$.

In Fig. 3, we sought to explore why three patients in the belinostat cohort had worse outcome than the historical controls. We investigated Cho/NAA abnormalities in these patients' pre-RT sMRI scan as Cho/NAA is a sensitive marker for regions at risk of recurrence [17]. The contours in the first column of Fig. 3 depict the margin of 2-fold or greater elevations in the Cho/NAA ratio (compared to the contralateral normal-appearing white matter). In the second column these are overlaid on the standard contrast enhanced MRI images to show the substantial difference between what is usually recognized as residual tumor and regions that predict tumor extent by Cho/NAA. This level of Cho/NAA elevation equates to a mean Z-score of 6.62 as reported previously (with $>99.999 \%$ confidence) [17]. In the third column, the margins for the 2 -fold or greater elevations in the Cho/NAA ratio are overlaid on FLAIR images. This shows somewhat greater but still incomplete concordance with extent of tumor predicted by the Cho/NAA ratio.

In row 3A, Cho/NAA elevation showed tumor spreading to the contralateral side through the corpus callosum, which wasn't apparent in pre-RT T1w-CE or FLAIR MRIs, and thus, was not treated within the radiation treatment plan. At recurrence, FLAIR hyperintensity had spread contralaterally in a similar direction to the Cho/NAA abnormality confirming tumor infiltration that was not treated during RT. In row 3B, the pre-RT Cho/NAA abnormality crosses the midline, which wasn't apparent in T1w-CE or even in FLAIR MRIs. Less than a year after RT, recurrence patterns with standard imaging confirmed findings in the pre-RT sMRI scan with both enhancement and FLAIR hyperintensity apparent within the pre-RT Cho/NAA contour. For this patient especially, the morphology of the T1w-CE and FLAIR lesions evolved throughout the follow-up period to approximate the morphology of the pre-RT Cho/NAA abnormality. In row $3 \mathrm{C}$, the pre-RT Cho/NAA abnormality spread widely anterior to the resection cavity, which was not visible in T1w-CE. Thus, this elevated Cho/NAA ratio abnormality was incompletely treated with high dose radiation. The recurrence pattern confirmed the findings in the pre-RT sMRI scan. The T1w-CE and FLAIR studies demonstrate increasing enhancement evolved throughout the follow-up period to approximate the morphology of the pre-RT Cho/NAA ratio abnormality. In summary, these three cases suggest that standard imaging underestimates the true extent of tumor infiltration, causing these patients to be undertreated and have a lower survival. Excluding these three patients, the median OS of belinostat cohort increases to over 30 months.

To gain a greater understanding of enhancing recurrence patterns relative the radiation dose distribution in study patients, we determined the actual dose received by the region of recurrence on a voxel-by-voxel basis. The minimum, maximum, and mean radiation dose to rGTV, the percentage of rGTV that occurred 
in PTV1, and the percentage of rGTV that occurred in PTV2 for each patient enrolled in the trial was determined and is listed in Supplemental Table 2. For the control versus the belinostat cohort patients, the minimum, maximum, and mean radiation dose to rGTV was on average 54.6 versus $45.0 \mathrm{~Gy}(p=0.20)$, 64.1 versus $57.9 \mathrm{~Gy}(\mathrm{p}=0.11)$, and 62.0 versus $51.5 \mathrm{~Gy}(\mathrm{p}=0.042)$, respectively. For the control versus the belinostat cohort patients, the mean percentage of rGTV within PTV1 and PTV2 was $99.3 \%$ versus $97.1 \%$ $(p=0.379)$ and $91.2 \%$ versus $85.5 \%(p=0.256)$, respectively. While all control patients had in-field failure as the site of first recurrence, only ten out of 13 belinostat patients failed in-field initially. Figure 4 illustrates this out-of-field failure in the three belinostat patients (cohort 2).

Treatment with belinostat given at a dose of $750 \mathrm{mg} / \mathrm{m}^{2} /$ day $\times 5$ days every 3 weeks during week 0 (preRT) as well as week 3 and week 6 of RT resulted in DLTs ( 2 with grade 4 thrombocytopenia and 1 with grade neutropenia) in 2 of 3 patients. However, after de-escalation of the belinostat dose to 500 $\mathrm{mg} / \mathrm{m}^{2} /$ day in the remaining cohort patients, no further DLTs were experienced. Significant toxicities (grade 3 or greater) that were judged to at least possibly be due to therapy for our control and belinostat cohorts are summarized on Table 1B. Overall, the addition of belinostat at the $500 \mathrm{mg} / \mathrm{m}^{2} /$ day dosing to concurrent RT/TMZ appears to be well tolerated.

\section{Discussion}

Here, we report the final results of a pilot study adding belinostat to concurrent RT and TMZ for patients with newly diagnosed GBMs [12]. Previously, the cohort receiving belinostat showed an improved 6month PFS compared to the control cohort ( $54 \%$ vs. $84 \%, p=0.073$ ). A speculated reason for the improved PFS at 6 months but not at 12 months is that belinostat was only given to subjects for a short duration throughout RT. HDACis like belinostat are known to be reversible drugs, and, epigenetic stress in tumors and the microenvironment may resume in the absence of HDACi resulting in a truncated effect. In our final analysis, the median overall survival was promising appearing slightly longer (by about 2.7 months) for the belinostat cohort. While the result is not statistically significant, this may be in part due to the small sample size. In a recent cooperative group trial (NRG-BN001) comparing dose-escalated to conventional dose RT for newly diagnosed GBM patients, patients on the experimental arm that received dose-escalated photon radiation had a median OS of 18.7 months [3]. The similarity in median OS benefit between the dose-escalated treatment arm of BN001 and our belinostat cohort points to the potential that belinostat may be a radiation sensitizer having a similar effect on patients comparable to dose escalation. In our trial, the voxel-based analysis of recurrence patterns comparing recurrent voxels to those in PTV2 indicates a potential shift of recurrences being more out-of-field suggesting that in-field control was improved due to the radiosensitizing effects of belinostat.

Pre-RT sMRI scans for a few representative belinostat patients reveal larger tumor extents (median volume difference was 6.2-folds between union of Cho/NAA $\geq 2 \mathrm{x}$ and residual tumor vs. residual tumor volume in all belinostat group patients) than were detected in standard imaging. Therefore, these portions of the lesion (as defined by Cho/NAA) were left undertreated. RT plans for all patients on the trial were guided by abnormality in T1w-CE and FLAIR only as per standard-of-care. Recurrence patterns confirm 
that pre-RT sMRI findings provide a possible reason for some in the belinostat cohort having an OS that was lower than that in historical controls.

The wide variability in OS suggests that some patients respond much more positively to a belinostat regimen while for others, the effect is minimal. While HDAC inhibitors are powerful epigenetic modulators, they are still target-specific drugs. They are hypothesized to work in the subgroup of patients whose tumorigenesis is driven by epigenetic modifications. This encourages investigation into whether certain subtypes of GBMs are more genetically predisposed to react positively to HDACi's like belinostat. Unfortunately, our sample size was too small to identify molecular subtypes that were responsive to belinostat. Interestingly, one patient with an IDH mutation who responded remarkably well to belinostat [13]. With this patient, there was not only a visible size reduction of metabolically active tumor volume but also the metabolic activity in the contralateral side of the brain was restored to healthy levels. This supports the observations reported by other groups that IDH1/2 mutations in GBM are associated with a fascinating link to 2-hydroxyglutarate (2-HG) accumulation representing an altered metabolite profile, which may have broad implications for both cancer epigenetics and clinical management of disease [19]. While it is difficult to run a clinical trial in GBM with IDH mutation in a single institution since the incident rate is low, it may be beneficial to run a multisite trial to determine if GBM with IDH mutation are unusually sensitive to belinostat.

Patients receiving belinostat on our study appear to have a different recurrence pattern than those in the control cohort. In particular, the mean radiation dose to the rGTV region was statistically significantly lower in the belinostat cohort than that in the control cohort (51.5 Gy vs $62.0 \mathrm{~Gy}, \mathrm{p}=0.042$ ) while other comparisons of the minimum and maximum dose to rGTV was similarly trending in the same direction. This suggests that concurrent belinostat may be delaying recurrences in regions that received higher radiation doses. The overlap between rGTV and PTV treatment zones was also lower in the belinostat cohort than that in the control cohort. Overall, these trends look promising for belinostat's activity in GBM especially in concert with full dose RT. This is further supported by the three patients in the belinostat cohort that had out-of-field recurrence as the first site of recurrence. In them the belinostat was unable to significantly impede recurrences when RT dose was inadequate.

Finally, the addition of belinostat to concurrent RT/TMZ at a dose of $750 \mathrm{mg} / \mathrm{m}^{2} /$ day $\times 5$ days every 3 weeks starting 1 week prior to RT did result in DLTs in 2 of 3 patients that completed this therapy. In one case, grade 4 thrombocytopenia first developed just prior to cycle 1 of adjuvant TMZ. In the other case, grade 4 thrombocytopenia and neutropenia did not develop until after receiving 3 cycles of adjuvant TMZ (more than 4 months following the last treatment with belinostat). While it is possible that these were spurious events as these hematologic toxicities can occur with TMZ alone, the lack of hematologic DLTs in our control cohort $(\mathrm{N}=13)$ and the remaining belinostat cohort $(\mathrm{N}=10)$ treated at $500 \mathrm{mg} / \mathrm{m}^{2} /$ day argues for implicating belinostat in these DLTs. Also, at the lower belinostat dose, no unusual toxicity was definitively noted. Thus, based on our limited experience, the dosing regimen of 3 cycles of belinostat at $500 \mathrm{mg} / \mathrm{m}^{2} /$ day $\times 5$ days every 3 weeks during concurrent RT/TMZ is well tolerated and should serve as a point of departure for dosing in future trials evaluating belinostat in combination with RT and TMZ. 
In this trial, belinostat was only administered during RT. Since an HDACi such as belinostat is a reversible drug and epigenetic modification-induced stress remains, it is certainly possible that extended use of belinostat during the maintenance period after radiation therapy may further improve outcomes in patients. This will require future testing to assess the safety and efficacy of belinostat when combined with adjuvant TMZ.

In summary, we have established that belinostat can be safely given with concurrent RT and TMZ and is trending towards improving outcomes in newly-diagnosed GBM patients. Because recurrence volumes in the control cohort had larger overlap with PTV2s (volume receiving $60 \mathrm{~Gy}$ ) than in the belinostat cohort, this suggests that belinostat has a better likelihood of delaying recurrence in those regions receiving 60 Gy. Trends in this study highlight the potential of belinostat, a BBB-penetrating $\mathrm{HDACi}$, as a synergistic therapeutic agent for GBM treatment.

\section{Declarations}

\section{Acknowledgements}

The authors would like to thank Spectrum Pharmaceuticals (Currently, Acrotech Biopharma LLC) for providing the investigational drug used in this clinical study; the staff at the Clinical Trials Office at the Winship Cancer Institute for their support and assistance with recruiting and managing the patients enrolled in the clinical study; and Dr. Andrew Maudsley and Mr. Sulaiman Sheriff at the University of Miami for their support with the EPSI sequence and MIDAS software framework.

Funding. This work is supported by National Institutes of Health grants, U01 CA172027 (PBB, JJO, HGS, HS) and U01 EB028145 (HS).

\section{Conflicts of interest/Competing interests. None}

Availability of data and material. Imaging data from patients in the study are available to other investigators upon request.

Code availability. No custom code was developed in this research. The recurrence pattern analysis software was reported previously and the original developer (ES) should be contacted for the code.

Authors' contributions. All authors contributed to the study conception and design. Material

preparation, data collection and analysis were performed by all authors. The first draft of the manuscript and tables/figures was prepared by Karen Xu and Karthik Ramesh and all authors commented on previous versions of the manuscript. All authors read and approved the final manuscript.

Ethics approval. The study was approved by the institutional review board (IRB). 
Consent to participate. Patients in this study provided informed consent for participation according to the institutional review board (IRB).

Consent for publication. Use of data for publication is included in the patient consent

\section{References}

1. Stupp R, Hegi ME, Mason WP, van den Bent MJ, Taphoorn MJ, Janzer RC, Ludwin SK, Allgeier A, Fisher B, Belanger K, Hau P, Brandes AA, Gijtenbeek J, Marosi C, Vecht CJ, Mokhtari K, Wesseling P, Villa S, Eisenhauer E, Gorlia T, Weller M, Lacombe D, Cairncross JG, Mirimanoff RO, European Organisation for R, Treatment of Cancer Brain Radiation Oncology T G, National Cancer Institute of Canada Clinical Trials G (2009) Effects of radiotherapy with concomitant and adjuvant temozolomide versus radiotherapy alone on survival in glioblastoma in a randomised phase III study: 5-year analysis of the EORTC-NCIC trial. Lancet Oncol 10: 459-466 doi:10.1016/S14702045(09)70025-7

2. Stupp R, Taillibert S, Kanner A, Read W, Steinberg D, Lhermitte B, Toms S, Idbaih A, Ahluwalia MS, Fink K, Di Meco F, Lieberman F, Zhu JJ, Stragliotto G, Tran D, Brem S, Hottinger A, Kirson ED, LavyShahaf G, Weinberg U, Kim CY, Paek SH, Nicholas G, Bruna J, Hirte H, Weller M, Palti Y, Hegi ME, Ram Z (2017) Effect of Tumor-Treating Fields Plus Maintenance Temozolomide vs Maintenance Temozolomide Alone on Survival in Patients With Glioblastoma: A Randomized Clinical Trial. JAMA 318:2306-2316. doi:10.1001/jama.2017.18718

3. Gondi V, Pugh S, Tsien C, Chenevert T, Gilbert M, Omuro A, Mcdonough J, Aldape K, Srinivasan A, Rogers C (2020) Radiotherapy (RT) Dose-intensification (DI) Using Intensity-modulated RT (IMRT) versus Standard-dose (SD) RT with Temozolomide (TMZ) in Newly Diagnosed Glioblastoma (GBM): Preliminary Results of NRG Oncology BN001. International Journal of Radiation Oncology Biology Physics 108:S22-S23

4. Stupp R, Mason WP, van den Bent MJ, Weller M, Fisher B, Taphoorn MJB, Belanger K, Brandes AA, Marosi C, Bogdahn U, Curschmann J, Janzer RC, Ludwin SK, Gorlia T, Allgeier A, Lacombe D, Cairncross JG, Eisenhauer E, Mirimanoff RO (2005) Radiotherapy plus Concomitant and Adjuvant Temozolomide for Glioblastoma. 352: 987-996 doi:10.1056/NEJMoa043330

5. Stupp R, Taillibert S, Kanner AA, Kesari S, Steinberg DM, Toms SA, Taylor LP, Lieberman F, Silvani A, Fink KL, Barnett GH, Zhu J-J, Henson JW, Engelhard HH, Chen TC, Tran DD, Sroubek J, Tran ND, Hottinger AF, Landolfi J, Desai R, Caroli M, Kew Y, Honnorat J, Idbaih A, Kirson ED, Weinberg U, Palti Y, Hegi ME, Ram Z (2015) Maintenance Therapy With Tumor-Treating Fields Plus Temozolomide vs Temozolomide Alone for Glioblastoma: A Randomized Clinical Trial. JAMA 314:2535-2543. doi:10.1001/jama.2015.16669 \%J JAMA

6. Gittleman H, Lim D, Kattan MW, Chakravarti A, Gilbert MR, Lassman AB, Lo SS, Machtay M, Sloan AE, Sulman EP, Tian D, Vogelbaum MA, Wang TJC, Penas-Prado M, Youssef E, Blumenthal DT, Zhang P, Mehta MP, Barnholtz-Sloan JS (2016) An independently validated nomogram for individualized 
estimation of survival among patients with newly diagnosed glioblastoma: NRG Oncology RTOG 0525 and 0825. Neuro-Oncology 19:669-677. doi:10.1093/neuonc/now208 \%J Neuro-Oncology

7. Was H, Krol SK, Rotili D, Mai A, Wojtas B, Kaminska B, Maleszewska M (2019) Histone deacetylase inhibitors exert anti-tumor effects on human adherent and stem-like glioma cells. Clin Epigenetics 11:11. doi:10.1186/s13148-018-0598-5

8. Mann BS, Johnson JR, Cohen MH, Justice R, Pazdur R (2007) FDA approval summary: vorinostat for treatment of advanced primary cutaneous T-cell lymphoma. Oncologist 12:1247-1252. doi:10.1634/theoncologist.12-10-1247

9. Hanson JE, La H, Plise E, Chen YH, Ding X, Hanania T, Sabath EV, Alexandrov V, Brunner D, Leahy E, Steiner P, Liu L, Scearce-Levie K, Zhou Q (2013) SAHA enhances synaptic function and plasticity in vitro but has limited brain availability in vivo and does not impact cognition. PLoS One 8:e69964. doi:10.1371/journal.pone.0069964

10. Wang C, Eessalu TE, Barth VN, Mitch CH, Wagner FF, Hong Y, Neelamegam R, Schroeder FA, Holson EB, Haggarty SJ, Hooker JM (2013) Design, synthesis, and evaluation of hydroxamic acid-based molecular probes for in vivo imaging of histone deacetylase (HDAC) in brain. Am J Nucl Med Mol Imaging 4:29-38

11. Lee HZ, Kwitkowski VE, Del Valle PL, Ricci MS, Saber H, Habtemariam BA, Bullock J, Bloomquist E, Li Shen Y, Chen XH, Brown J, Mehrotra N, Dorff S, Charlab R, Kane RC, Kaminskas E, Justice R, Farrell AT, Pazdur R (2015) FDA Approval: Belinostat for the Treatment of Patients with Relapsed or Refractory Peripheral T-cell Lymphoma. Clin Cancer Res 21:2666-2670. doi:10.1158/10780432.CCR-14-3119

12. Gurbani SS, Yoon Y, Weinberg BD, Salgado E, Press RH, Cordova JS, Ramesh KK, Liang Z, Velazquez Vega J, Voloschin A, Olson JJ, Schreibmann E, Shim H, Shu HG (2019) Assessing Treatment Response of Glioblastoma to an HDAC Inhibitor Using Whole-Brain Spectroscopic MRI. Tomography 5:53-60. doi:10.18383/j.tom.2018.00031

13. Gurbani SS, Weinberg BD, Salgado E, Voloschin A, Velazquez Vega JE, Olson JJ, Shu H-KG, Shim H (2020) Remarkable response of a patient with secondary glioblastoma to a histone deacetylase inhibitor. Oxford Medical Case Reports 2020 doi:10.1093/omcr/omaa006

14. Sabati M, Sheriff S, Gu M, Wei J, Zhu H, Barker PB, Spielman DM, Alger JR, Maudsley AA (2015) Multivendor implementation and comparison of volumetric whole-brain echo-planar MR spectroscopic imaging. Magn Reson Med 74:1209-1220. doi:10.1002/mrm.25510

15. Maudsley AA, Darkazanli A, Alger JR, Hall LO, Schuff N, Studholme C, Yu Y, Ebel A, Frew A, Goldgof D, Gu Y, Pagare R, Roulsseau F, Sivasankaran K, Soher BJ, Weber P, Young K, Zhu X (2006) Comprehensive processing, display and analysis for in vivo MR spectroscopic imaging. NMR Biomed 19:492-503. doi:10.1002/nbm.1025

16. Maudsley AA, Domenig C, Govind V, Darkazanli A, Studholme C, Arheart K, Bloomer C (2009) Mapping of Brain Metabolite Distributions by Volumetric Proton MR Spectroscopic Imaging (MRSI). Magn Reson Med 61:548-559. doi:10.1002/mrm.21875 
17. Cordova JS, Shu H-KG, Liang Z, Gurbani SS, Cooper LAD, Holder CA, Olson JJ, Kairdolf B, Schreibmann E, Neill SG, Hadjipanayis CG, Shim H (2016) Whole-brain spectroscopic MRI biomarkers identify infiltrating margins in glioblastoma patients. Neuro-Oncology 18:1180-1189.

doi:10.1093/neuonc/now036 \%J Neuro-Oncology

18. Gurbani S, Weinberg B, Cooper L, Mellon E, Schreibmann E, Sheriff S, Maudsley A, Goryawala M, Shu HK, Shim H (2019) The Brain Imaging Collaboration Suite (BrICS): A Cloud Platform for Integrating Whole-Brain Spectroscopic MRI into the Radiation Therapy Planning Workflow. Tomography 5:184191. doi:10.18383/j.tom.2018.00028

19. Prensner JR, Chinnaiyan AM (2011) Metabolism unhinged: IDH mutations in cancer. Nat Med 17:291-293. doi:10.1038/nm0311-291

\section{Tables}

Table 1. (A) Summary statistics for both cohorts; (B) Tabulation of significant toxicities ( $\geq$ grade 3 ) in all patients. *All occurrences were at $750 \mathrm{mg} / \mathrm{m}^{2} /$ day dosing.

A

\begin{tabular}{lll} 
& Control & Belinostat \\
\hline Number of Patients & 13 & 13 \\
\hline Age & $58.5 \pm 11.1$ & $51.2 \pm 11.6$ \\
\hline IDH1 Mutation & $1(7.7 \%)$ & $1(7.7 \%)$ \\
\hline MGMT Methylated & $4(30.8 \%)$ & $4(30.8 \%)$
\end{tabular}

B

\begin{tabular}{llll} 
Toxicities & Grade & Control cohort & Belinostat cohort \\
\hline Thrombocytopenia & 4 & $0 / 13$ & $2 / 13^{\star}$ \\
\hline Neutropenia & 4 & $0 / 13$ & $1 / 13^{\star}$ \\
\hline Lymphopenia & 3 & $3 / 13$ & $1 / 13^{\star}$ \\
\hline Constipation & 3 & $1 / 13$ & $1 / 13$ \\
\hline Fatigue & 3 & $1 / 13$ & $1 / 13$ \\
\hline Confusion & 3 & $1 / 13$ & $0 / 13$
\end{tabular}

\section{Figures}




\begin{tabular}{|c|c|c|c|c|c|c|c|c|c|c|c|c|c|c|c|c|c|}
\hline Week & $\begin{array}{l}\text { Prior to } \\
\text { enrollment }\end{array}$ & 0 & 1 & 2 & 3 & 4 & 5 & 6 & & 11 & 12 & 13 & 14 & & $6 \mathrm{mos}$ & & $12 \mathrm{mos}$ \\
\hline $\begin{array}{r}\text { Surgical } \\
\text { Resection }\end{array}$ & & & & & & & & & \multirow{6}{*}{ 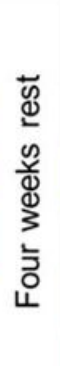 } & & & & & \multirow{5}{*}{ 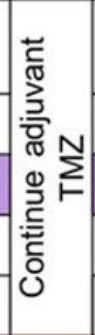 } & & \multirow{5}{*}{ 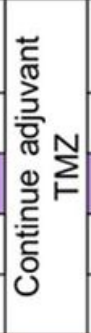 } & \\
\hline Belinostat & & & & & & & & & & & & & & & & & \\
\hline TMZ & & & \multicolumn{6}{|c|}{ Cycle 1 (42 days) } & & \multicolumn{4}{|c|}{ Cycle $2+$ (28 days) } & & & & \\
\hline Radiation & & & \multicolumn{6}{|c|}{30 fractions } & & & & & & & & & \\
\hline IDS-SR & & & & & & & & & & & & & & & & & \\
\hline Clinical Imaging & & & & & & & & & & \multicolumn{8}{|c|}{$\sim$ every 2 mos after RT } \\
\hline
\end{tabular}

\section{Figure 1}

One-year timeline of chemotherapy, intravenous belinostat, and radiation for patients in NCT02137759. 


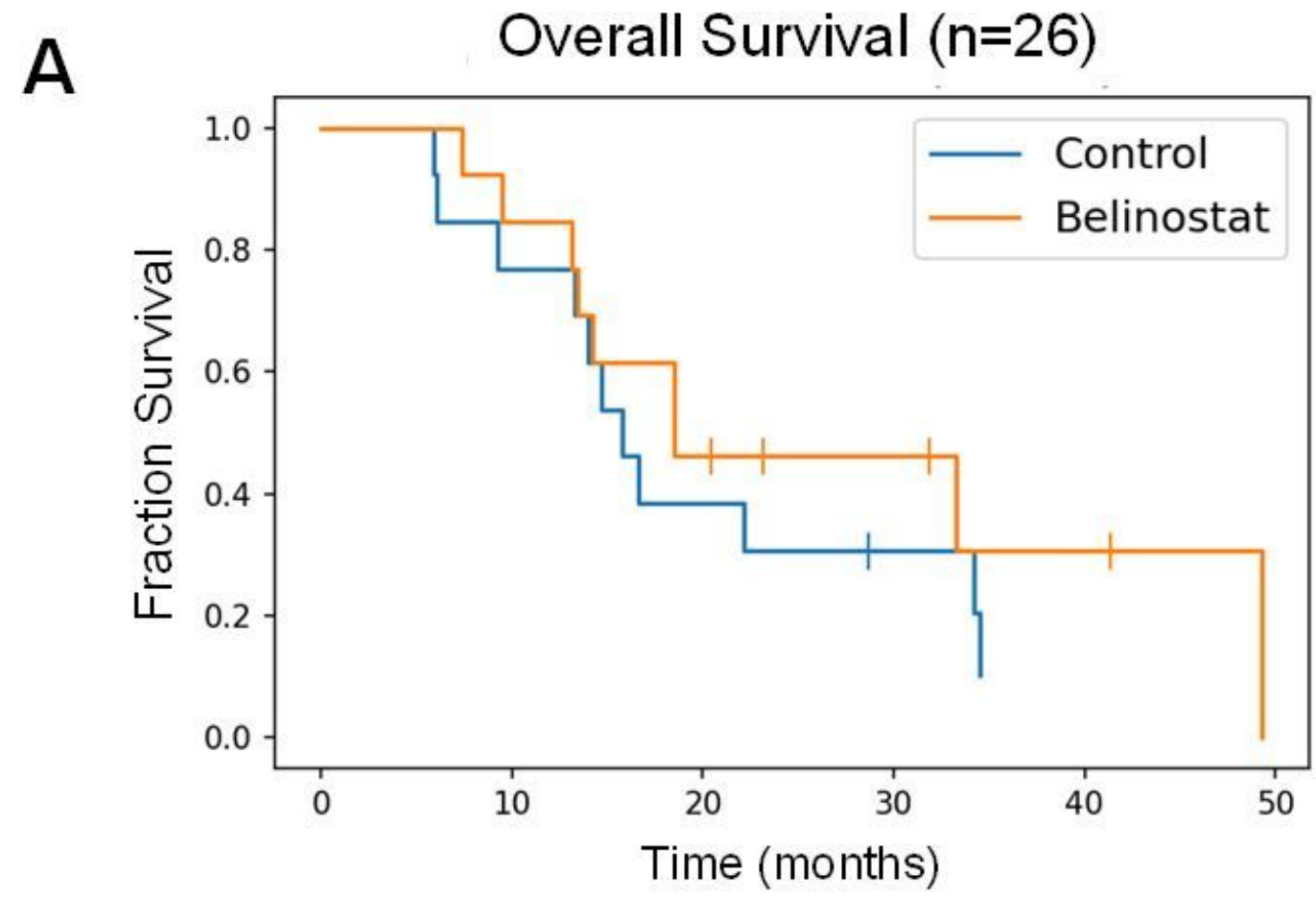

B

Progression Free Survival $(n=26)$

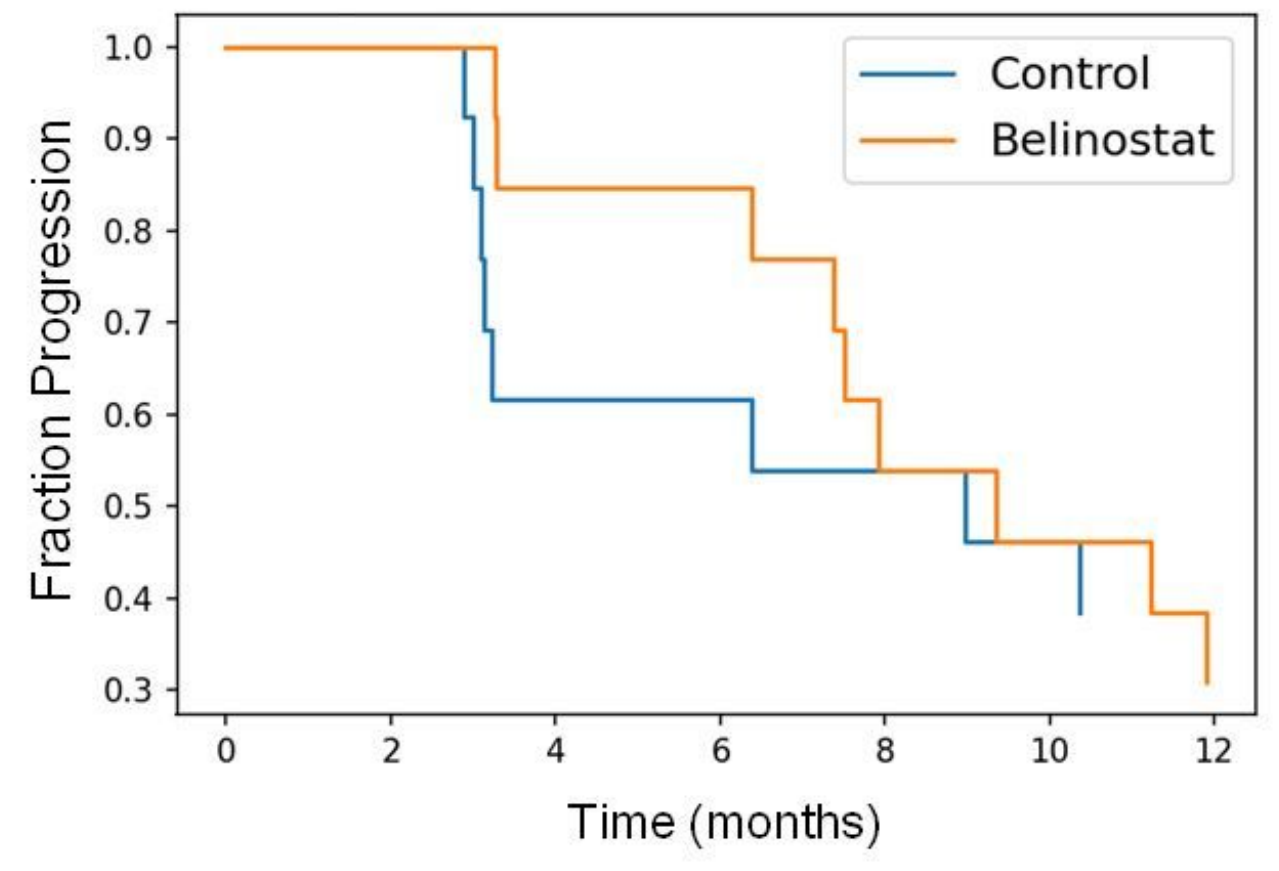

Figure 2

Overall Survival (OS) Kaplan-Meier Survival Curves (A) and Progression Free Survival (PFS) (bottom) for all patients. The median OS for the control and belinostat cohorts was 15.8 and 18.5 months, respectively $(p=0.53)$, for all patients. The median PFS for control and belinostat cohorts was 9.0 months and 9.3 months, respectively $(\mathrm{p}=0.75)$. 


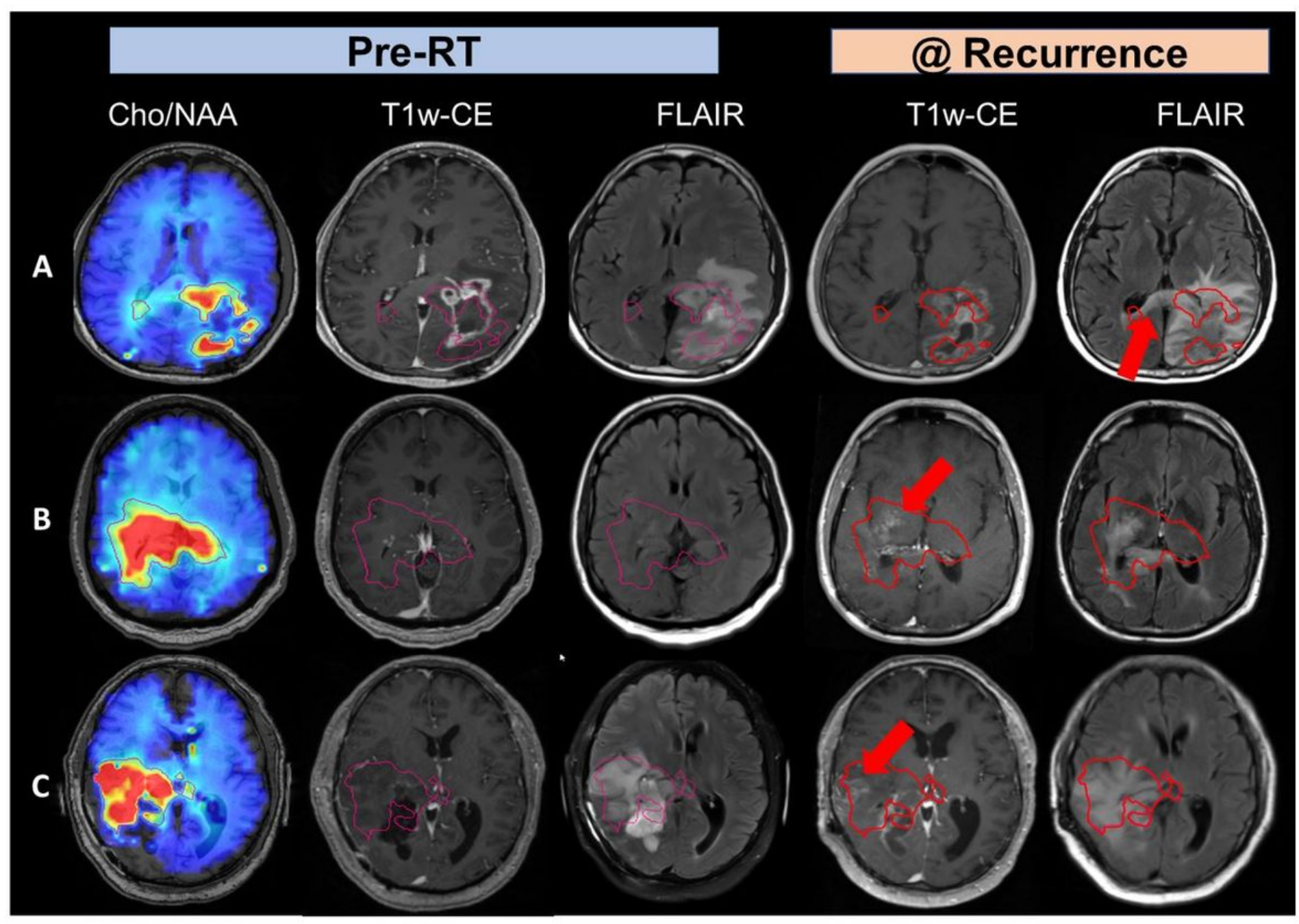

Figure 3

Pre-RT sMRI scans suggest out-of-field recurrence in the belinostat cohort had tumor infiltration beyond what are shown in standard imaging. A) This patient had two-fold Cho/NAA elevation that had spread contralaterally. While pre-RT standard imaging failed to detect this, FLAIR hyperintensity at recurrence mimics the direction of tumor infiltration detected by pre-RT sMRI. B) The second patient had tumor infiltration across the midline that wasn't detected by pre-RT standard imaging. Lesions at recurrence in both T1w-CE and FLAIR MRIs confirm Cho/NAA abnormalities in the pre-RT sMRI scan, suggesting standard imaging underestimated the extent of tumor infiltration. C) This patient had a large lesion of metabolically active tumor that was not shown in T1w-CE, thus, undertreated, which became apparent at recurrence. 


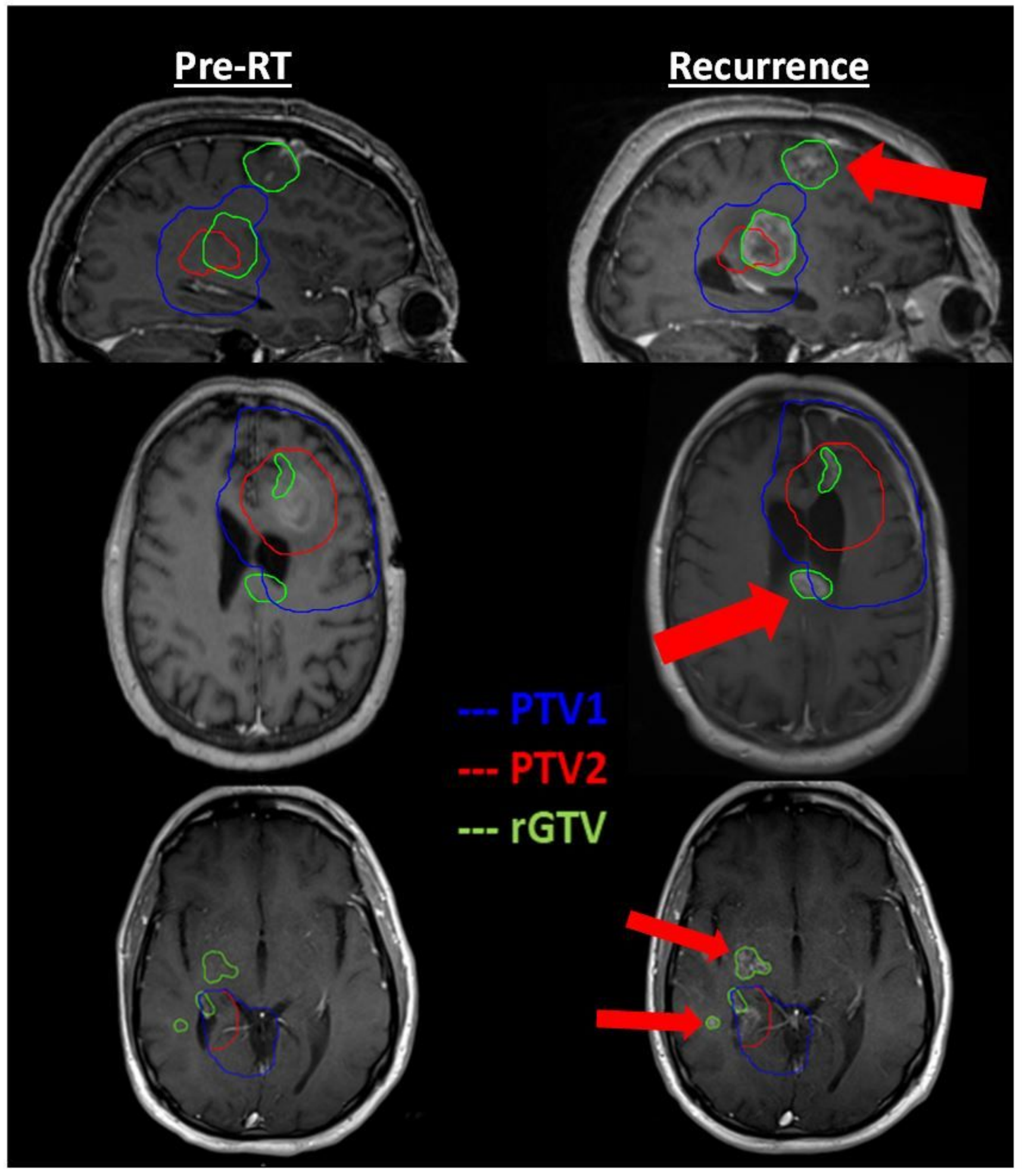

Figure 4

T1 post-contrast imaging in 3 patients, by horizontal row, with out of field recurrence in cohort 2. For each patient, the rGTV contour encompasses lesion that has spread outside the extent of PTV1 or PTV2.

\section{Supplementary Files}


This is a list of supplementary files associated with this preprint. Click to download.

- Supplebelinostat2021.docx 